\title{
Radial pseudoaneurysm in elderly: a rare event with undefined therapeutical approach. A case report and literature review
}

\author{
Emanuele Gallinoro, Francesco Natale, Saverio D’Elia, Paolo Golino, Giovanni Cimmino \\ Department of Translational Medical Sciences, University of Campania "Luigi Vanvitelli", Cardiology Unit "Vanvitelli", \\ Monaldi Hospital, Naples, Italy
}

\begin{abstract}
Radial artery pseudoaneurysm (RAP) after cardiac catheterization in elderly patients is a rare complication. Clinical manifestations are pain, swelling and haematoma of the harm. The diagnosis is made through doppler ultrasonography, but the best therapeutical option is still matter of debate. Traditionally, surgical treatment has been considered the gold standard but new and less invasive strategies have been recently proposed: ultrasound-guided compression and local injection of thrombin. In this report we describe the unique case of an 84-year-old female patient who developed radial artery pseudoaneurysm after a failed radial artery access for cardiac catheterization. Finally, the pseudoaneurysm
\end{abstract}

Correspondence: Giovanni Cimmino, Department of Translational Medical Sciences, University of Campania "Luigi Vanvitelli". Cardiology Unit "Vanvitelli", Monaldi Hospital, via Leonardo Bianchi 1,80131 Naples, Italy. E-mail: giovanni.cimmino@unicampania.it

Contributions: EG, FN, SDE, substantial contributions to the conception of the work, acquisition and analysis of data, drafting of the work, final approval of the version to be published, agreement to be accountable for all aspects of the work in ensuring that questions related to the accuracy or integrity of any part of the work are appropriately investigated and resolved; GC, PG, substantial contributions to the design of the work and interpretation of data, manuscript revision for important intellectual content, final approval of the version to be published, agreement to be accountable for all aspects of the work in ensuring that questions related to the accuracy or integrity of any part of the work are appropriately investigated and resolved.

Conflict of interest: the authors certify that there is no conflict of interest with any financial organization regarding the material discussed in the manuscript

Key words: Radial artery pseudoaneurysm; cardiac catheterization; yin-yang sign.

Received for publication: 9 June 2019

Accepted for publication: 5 July 2019.

${ }^{\circ}$ Copyright: the Author(s), 2019

Licensee PAGEPress, Italy

Monaldi Archives for Chest Disease 2019; 89:1109

doi: 10.4081/monaldi.2019.1109

This article is distributed under the terms of the Creative Commons Attribution Noncommercial License (by-nc 4.0) which permits any noncommercial use, distribution, and reproduction in any medium, provided the original author(s) and source are credited. was successfully treated by surgical approach as several attempts of local compression failed. We aimed also at reviewing the treatment options of RAP in elderly patients ( $>75$ years old) and the safety/effectiveness reported in literature.

\section{Introduction}

Trans-radial access (TRA) for cardiac catheterization has become the preferred access site over the last decades because of the lower incidence of major bleeding and access site complications compared to the historical trans-femoral approach [1]. Also in patients with acute coronary syndrome, TRA has shown to reduce adverse clinical events, through a reduction in major bleeding and all-cause mortality [1]. However, potential complications have been described also for TRA [2] and, despite of being rare and unique, they can be part of our clinical practice, thus it is important to set up their management and prevention.

Common complications are radial artery occlusion (incidence rate $1-38 \%$ ) and radial artery spasm [2]. Less common complications are forearm haematoma, compartment syndrome, radial artery perforation, arteriovenous fistula formation, pseudoaneurysm, nerve damage, granuloma formation, and catheter entrapment [2]. Radial artery pseudoaneurysm (RAP) is a rare complication, whose incidence has been shown to be less than $0.1 \%$ in other case reports [3]. Possible risk factors for the development of post-procedural pseudoaneurysm are inadequate compression and use of anticoagulants [3]. Although no clinical trials exist analysing the best treatment for iatrogenic RAP, several therapeutic strategies have been described in literature: surgical approach, local compression and local thrombin injection [4]. In this article we describe a unique case of a RAP in elderly patient after a failed radial artery access for coronary angiography, the failure of two consecutive compressions and the successful treatment by surgical repair under local anaesthesia. Then we have reviewed the literature about incidence and treatment of RAP after cardiac catheterization in elderly (>75-year-old) patients.

\section{Case Report}

An 84-year-old female patient with a history of breast cancer already treated with chemotherapy and surgery, chronic hepatitis$\mathrm{C}$-virus infection, hypertension and permanent atrial fibrillation was admitted to the department of emergency medicine for acute dyspnoea. Echocardiography revealed severe aortic stenosis [lowflow, low-gradient, mean gradient $29 \mathrm{mmHg}$, aortic valve area 
(AVA) $0.28 \mathrm{~cm}^{2}$, stroke volume $25 \mathrm{ml} / \mathrm{m}^{2}$, ejection fraction (EF) $42 \%$ ]. Because of the high risk for surgery, as for the heart team discussion, the patient was selected for transcatheter aortic valve implantation (TAVI) procedure. Before TAVI a coronary angiography was performed. As for standard procedure, right radial artery approach was pursued. Despite of a good pulse and 6 to 7 attempts, transradial access was not achieved with inability to advance the wire by the operator. As a consequence, left femoral artery access was employed. Percutaneous coronary intervention (PCI) with multiple drug eluting stent implantation on right coronary artery, circumflex artery and anterior interventricular artery was performed. A local haemostasis with local compression was applied and immobilization of the leg was prescribed for $24 \mathrm{~h}$, as per standard procedure. On the radial artery a local haemostasis was achieved by using TR-band that was removed after $3 \mathrm{~h}$, as per protocol. The day after, a local swelling of the right arm was observed (Figure 1) and, as per team discussion, a local compressive bandage not adversely affecting arterial flow was applied from the elbow to the wrist. A daily evaluation of the arm was performed. Three days after PCI the patient underwent TAVI procedure through right femoral artery. At day 4 after cardiac catheterization the bandage was removed for re-evaluation, but a visible oedema of the arm was still present. Patient was also experiencing a painful haematoma on the third distal of the volar side of the right arm, despite of the radial site was not suitable to perform cardiac catheterization. At the time of the coronary procedure the ongoing therapy was acetylsalicylic acid (ASA) $100 \mathrm{mg} /$ day, Clopidogrel $75 \mathrm{mg}$, Bisoprolol $2.5 \mathrm{mg} /$ day, Pantoprazole $40 \mathrm{mg} /$ day and Enoxaparin $6000 \mathrm{UI} /$ day.

The ultrasonography evaluation showed a hypoechoic cystic structure $(20 \times 20 \mathrm{~mm})$ nearby the third distal of the radial artery. The colour doppler analysis showed blood flow in the cystic mass (supplied by the radial artery) with a swirling motion pattern, known as yin-yang sign, as shown in Figure 2. The continuous wave doppler aligned on the neck of the cystic mass showed the toand-fro pattern, thus the diagnosis of RAP was made (Figure 2). Two attempts of treatment by local ultrasound-guided compression applied for $30 \mathrm{~min}$ were performed, both of them failed. Then, a local compressive bandage was applied for $24 \mathrm{~h}$ but, at the end of this time, the pseudoaneurysm was still supplied by the radial artery. At this point, as for team discussion, surgical treatment was

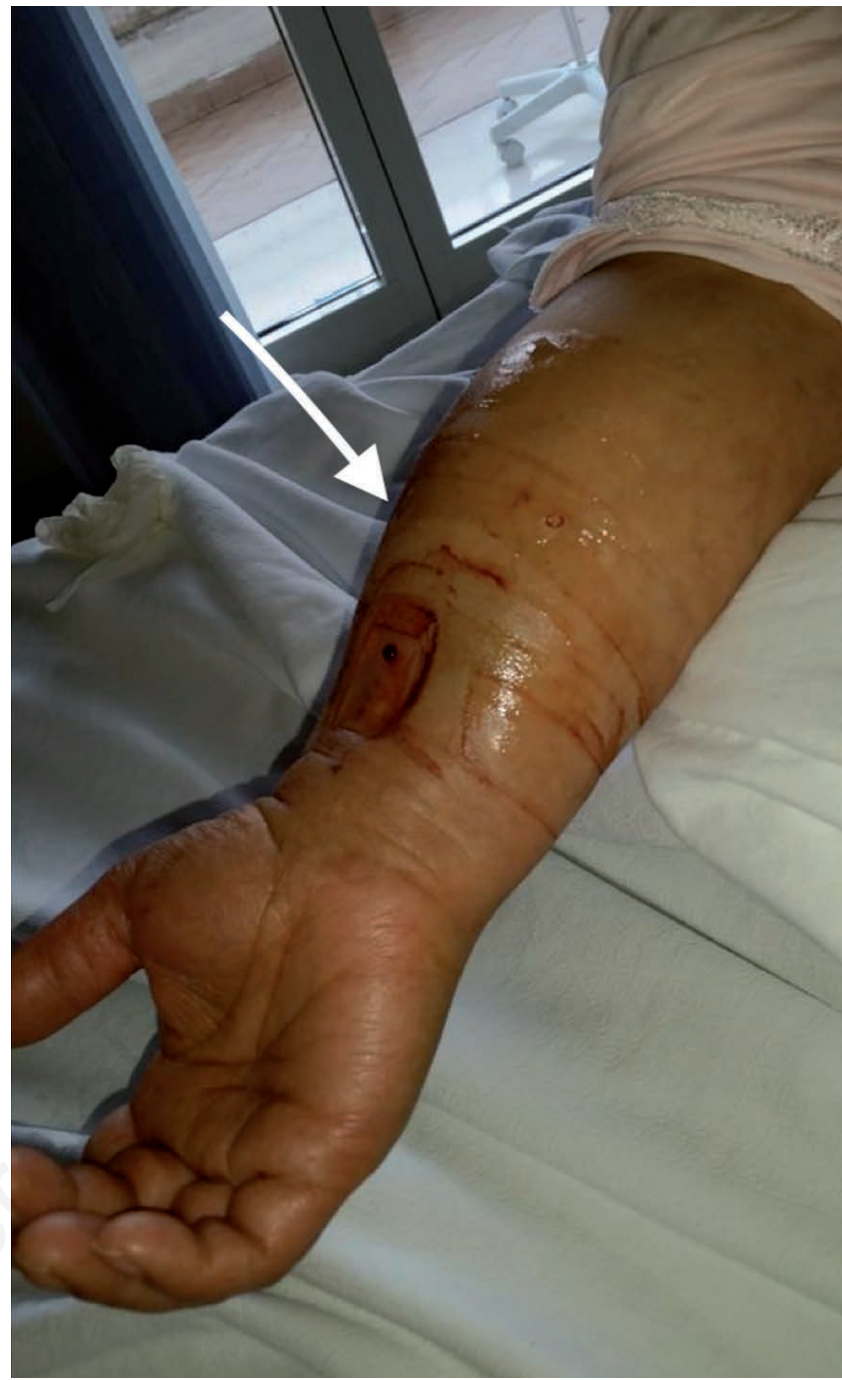

Figure 1. Painful haematoma covering the volar side of the right arm. Site of puncture and TR-band application are clearly visible. Arrow indicates pseudoaneurysm.

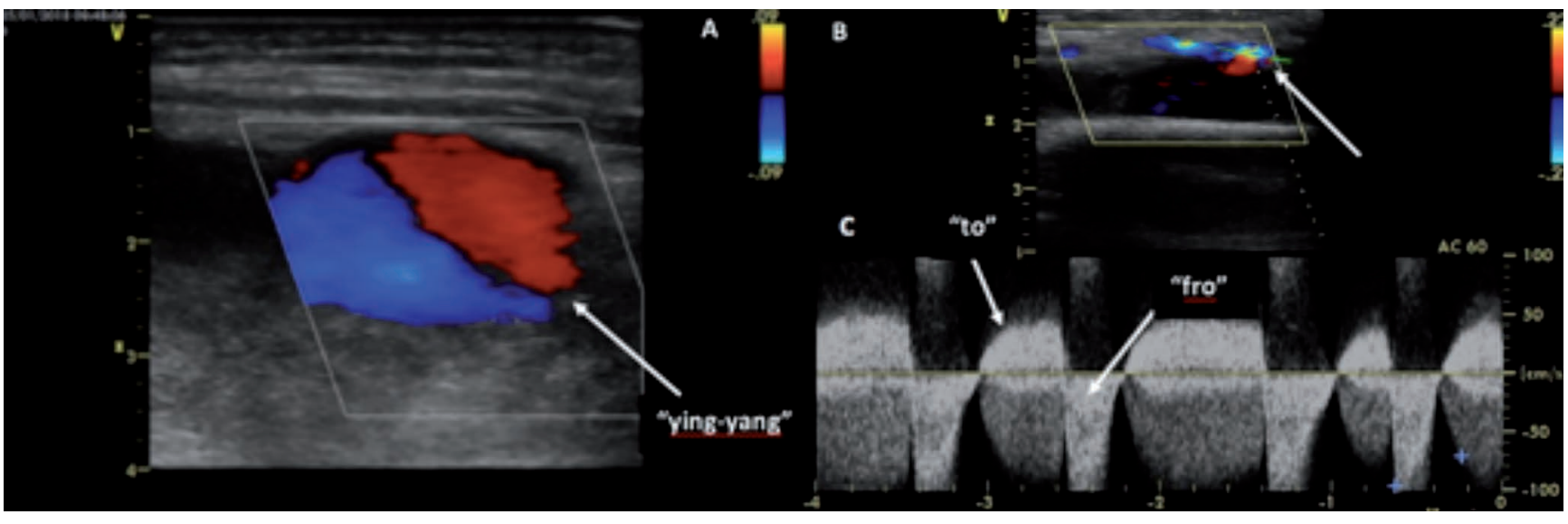

Figure 2. Ultrasound colour doppler image of the radial artery pseudoaneurysm; on the left is visible the typical yin-yang sign. On the right the to-and-fro pattern at power doppler analysis. The arrow indicates the neck of the pseudoaneurysm. 
proposed. A local anaesthesia was made and a longitudinal incision at the third distal of the arm was performed to expose the vascular lesion that was sutured with polypropylene $7 / 0$ and the haematoma drained. Time of operation was 50 min with the complete RAP resolution. No complications were recorded after the surgical procedure. The patient was discharged at days 7 after surgery on regular oral ASA $100 \mathrm{mg}$ and Clopidogrel $75 \mathrm{mg}$ as antiplatelet therapy and Apixaban $2.5 \mathrm{mg}$ bid as anticoagulation treatment. Moreover, a final echocardiographic control was performed with no RAP reported. Three month later a follow-up visit was scheduled reporting absence of pain and oedema with a complete recovery of arm and hand function.

\section{Discussion}

Radial pseudoaneurysm is a really rare complication after cardiac catheterization [5]. Ultrasonography is the diagnostic tool of choice [6]. Using a linear (high frequency) probe with a grey scale is possible to detect the area of pseudoaneurysm; then, by using colour doppler evaluation the yin-yang sign is detectable from blood entering the sac during systole and exiting during diastole. At the power doppler evaluation, the to-and-fro pattern is reported as typical sign [6].

As RAP is a rare complication, there is still no evidence about the best therapeutic strategy. Possible risk factors for its development are inadequate compression ad use of anticoagulation treatments. Age seems to be not a risk factor. However, as reported in our case and in others, the effectiveness of the treatment options can be influenced by the age [2].

Several strategies have been described in literature for RAP treatment [7]. To date, surgical approach is considered the gold standard. It usually consists in excision of RAP followed by radial artery repair. Ultrasound guided compression or local compression using bandage or specific devices (such as TR-band) has been shown to be effective in some cases [4]. However, it has to be considered that local prolonged compression of radial artery may result in occlusion or even rupture of the outer wall. Ultrasound-guided local thrombin injection appears to be a possible alternative strategy as it has already showed to be an effective treatment for femoral iatrogenic pseudoaneurysm [4]. A possible complication is radial thrombosis.
In literature has been described also a case of endovascular treatment of RAP by keeping a sheath in the radial artery for $8 \mathrm{~h}$ (attached to a pressurized heparinized saline flush using a standard arterial line setup) covering the neck of the RAP [7].

For elderly patients ( $>75$-years old) other reports have been already published in literature. Zegrí et al. described four patients over 75 years of age affected by post cardiac catheterization RAP [4]. All patients were initially treated with local compression recording two complete resolutions of the RAP (one at second attempt) out of four. The other two cases were successfully treated with local thrombin injection [4]. However, two RAP ruptures and one radial artery thrombosis were also reported [4]. Nazer et al. described a complete resolution of a RAP at the second attempt of local compression as the first failed [8], while Hamid et al. described reported a successful surgical repair in two octogenarians [9]. Moreover, Bachani et al. reported a case of RAP unsuccessfully treated with local compression followed a resolutive surgical intervention [10]. Other cases of RAP in elderly patients have been reported $[3,5,11]$ : only one was successfully treated with local compression [5] while the others $[3,11]$ underwent surgical treatment. US-guided local thrombin injection might be another possible option [12] as shown by Mohamed et al. described in an octogenarian. Reported cases, patients' characteristics, treatment options, and complications are summarized in Table 1.

By analysing the published cases, local compression seems to be the easiest and the cheapest approach for RAP treatment even if it appears to be not as effective as other treatments, especially in elderly patients. Anatomy of RAP can affect the success of compression treatment: in our case the neck of the pseudoaneurysm was really short and, as the mass appears to be in strict continuation with the artery, local compression could not lead to the complete occlusion of the neck. A long neck, instead, running through subcutaneous tissues could be easily closed by external compression (as the pressure is transmitted to the subcutaneous tissues and then to the neck). Ultrasound-guided thrombin injection has shown to be an effective procedure for femoral pseudoaneurysm [12] and maybe it would be a valid approach also for RAP in elderly patient; however, it is important to note that this therapy is still unlicensed and may lead to potentially serious complications such as artery thrombosis. Moreover, it remains a discrepancy on the standardised dose of thrombin required to achieve successful thrombosis of the aneurismal sac. Further studies are warranted to assess effec-

Table 1. Summary the published cases with patients' characteristics, therapeutical approach, complication and effectiveness.

\begin{tabular}{|c|c|c|c|c|c|c|c|c|}
\hline \multirow[t]{2}{*}{ Author } & \multirow{2}{*}{$\begin{array}{c}\text { Number } \\
\text { of patients } \\
\text { over } 75 \text { years }\end{array}$} & \multirow{2}{*}{$\begin{array}{c}\text { Age of each } \\
\text { patient } \\
\text { (yrs) }\end{array}$} & \multicolumn{2}{|c|}{ Compression } & \multicolumn{2}{|c|}{ Thrombin injection } & \multicolumn{2}{|c|}{ Surgery } \\
\hline & & & $\begin{array}{l}\text { Successful } \\
\text { (n) }\end{array}$ & $\begin{array}{c}\text { Complication } \\
\text { (n) }\end{array}$ & $\begin{array}{l}\text { Successful } \\
\text { (n) }\end{array}$ & $\begin{array}{l}\text { Complication } \\
\text { (n) }\end{array}$ & $\begin{array}{l}\text { Successful } \\
\text { (n) }\end{array}$ & $\begin{array}{c}\text { Complication } \\
\text { (n) }\end{array}$ \\
\hline Zegrí & 4 & $76,79,88,81$ & 2 & 2 & 2 & 1 & - & - \\
\hline Bachani & 1 & 79 & 1 & 1 & - & - & 1 & - \\
\hline Bath & 1 & 80 & - & - & - & - & 1 & 0 \\
\hline Nazer & 1 & 77 & $1(\mathrm{x} 2)$ & 0 & - & - & - & - \\
\hline Mohamed & 1 & 85 & - & - & 1 & 0 & - & - \\
\hline Suchòn & 1 & 85 & 1 & 0 & - & - & - & - \\
\hline Hamid & 2 & 80,83 & - & - & - & - & 2 & 0 \\
\hline Collins & 1 & 82 & - & - & - & - & 1 & 0 \\
\hline Tosti & 2 & 72,83 & - & - & - & - & 2 & 0 \\
\hline Gallinoro & 1 & 84 & 0 & 0 & - & - & 1 & 0 \\
\hline
\end{tabular}


tiveness of this procedure. Surgical treatment, consisting in pseudoaneurysm drainage or excision followed by radial artery wall repair under local anaesthesia appears to be still a safety and effective procedure for RAP treatment even in elderly patients.

\section{Conclusions}

Radial artery pseudoaneurysm is a rare complication after cardiac catheterization with undefined therapeutical strategy. Radial access together with the insertion of wires and catheters are the main factors involved in pseudoaneurysm formation. To our knowledge, this is the first reported case of RAP in an elderly patient resulted only from an attempt of a radial access, as none wires or catheters were inserted. Diagnosis was made by clinical examination and confirmed through ultrasound evaluation. Surgical approach has been the only effective strategy. Alternative treatment options are described in literature with unclear safety and effectiveness. This case, together with other reported in literature may suggest, by the statistical point of view, a possible therapeutical management.

\section{References}

1. Valgimigli M, Gagnor A, Calabro P, et al. Radial versus femoral access in patients with acute coronary syndromes undergoing invasive management: a randomised multicentre trial. Lancet 2015;385:2465-76.

2. Arora V, Patel M, Shroff AR. Complications of transradial car- diac catheterization and management. Interv Cardiol Clin 2015;4:193-202.

3. Tosti R, Özkan S, Schainfeld RM, Eberlin KR. Radial artery pseudoaneurysm. J Hand Surg Am 2017;42:295.e1.

4. Zegrí I, García-Touchard A, Cuenca S, et al. Radial artery pseudoaneurysm following cardiac catheterization: clinical features and nonsurgical treatment results. Rev Esp Cardiol (Engl Ed) 2015;68:349-51.

5. Suchoń E, Jąkała J, Dykla D, et al. Radial artery pseudoaneurysm as an extremely rare complication associated with transradial catheterisation. Kardiol Pol 2013;71:542.

6. Mahmoud MZ, Al-Saadi M, Abuderman A, et al. "To-and-fro" waveform in the diagnosis of arterial pseudoaneurysms. World J Radiol 2015;7:89-99.

7. Babunashvili AM, Pancholy SB, Kartashov DS. New technique for treatment of postcatheterization radial artery pseudoaneurysm. Catheter Cardiovasc Interv 2017;89:393-8.

8. Nazer B, Boyle A. Treatment of recurrent radial artery pseudoaneurysms by prolonged mechanical compression. J Invasive Cardiol 2013;25:358-9.

9. Hamid T, Harper L, McDonald J. Radial artery pseudoaneurysm following coronary angiography in two octogenarians. Exp Clin Cardiol. 2012;17:260-2.

10. Bachani N, Lokhandwala Y, Vajifdar B, Panicker GK. Radial artery pseudoaneurysm. J Indian Coll Cardiol 2016;6:142-4.

11. Bhat $\mathrm{T}$, Bhat $\mathrm{H}$, Teli $\mathrm{S}$, et al. Pseudoaneurysm a rare complication of transradial cardiac catheterization: a case report. Vascular 2013;21:331-4.

12. Mohamed MO, Saif M, Townend JN, Khan SQ. Successful treatment of a radial artery pseudoaneurysm in an octogenarian. BMJ Case Rep 2015. doi: 10.1136/bcr-2015-211513. 